\title{
Living with Constraints
}

\author{
Alan K. Mackworth ${ }^{\star}$ \\ Department of Computer Science \\ University of British Columbia \\ Vancouver, B.C., Canada V6T 1Z4
}

\begin{abstract}
In order to thrive, an agent must satisfy dynamic constraints deriving from four sources: its internal structure, its goals and preferences, its external environment and the coupling between its internal and external worlds. The life of any agent who does not respect those constraints will be out of balance. Based on this framing of the problem of agent design, I shall give four perspectives on the theme of living with constraints, beginning with a theory of constraint-based agent design and a corresponding experiment in robot architecture. Second, I shall touch briefly on a personal historical note, having lived with the evolving concept of the pivotal role of constraints throughout my research life. Third, I shall outline our work on the design of two assistive technology prototypes for people with physical and mental disabilities, who are living with significant additional constraints. Finally, I shall suggest our collective failure to recognize, satisfy and live with various constraints could explain why many of the worlds we live in seem to be out of kilter. This approach hints at ways to restore the balance. Some of the work discussed is joint with Jim Little, Alex Mihailidis, Pinar Muyan-Ozçelik, Robert St-Aubin, Pooja Viswanathan, Suling Yang, and Ying Zhang.
\end{abstract}

* Canada Research Chair in Artificial Intelligence. 